This paper is published in the open archive of Mid Sweden University

DIVA http://miun.diva-portal.org

by permission of the publisher

Marcus Barkowsky, Sylvain Tourancheau, Kjell Brunnström, Kun Wang, and Borje Andrén, "55.3: Crosstalk Measurements of Shutter Glasses 3D Displays", SID Symposium Digest 42, 812 (2011).

http://dx.doi.org/10.1889/1.3621454

(C) Copyright 2011 Society for Information Display (SID). One print or electronic copy may be made for personal use only. Systematic electronic or print reproduction and distribution, duplication of any material in this paper for a fee or for commercial purposes, or modification of the content of the paper are prohibited. 


\title{
55.3: Crosstalk Measurements of Shutter Glasses 3D Displays
}

\author{
Marcus Barkowsky', Sylvain Tourancheau'², Kjell Brunnström³ ${ }^{3}$ Kun Wang ${ }^{2,3}$, Börje Andrén ${ }^{3}$ \\ ${ }^{1}$ IRCCyN, Polytech'Nantes, University of Nantes, 44306 Nantes, France \\ ${ }^{2}$ Dept. of Information Technology and Media, Mid Sweden University (MIUN), 85170 Sundsvall, Sweden \\ ${ }^{3}$ NetLab: IPTV, Video and Display Quality, Acreo AB, 16440 Kista, Sweden
}

\begin{abstract}
Crosstalk is probably one of the main perceptual factors contributing to perceived image quality and visual comfort. The Video Quality Experts Group (VQEG) within their $3 D$ video quality project is specifying a practical measurement procedure that will produce consistent results across laboratories. This paper is part of that effort. Two different method of measuring cross talk on shutter glasses stereo displays have been studied. One is based on time average luminance measurements and the other on temporal measurements. The results show that crosstalk is roughly $0.5 \%$ but that there are differences in the crosstalk between the two eyes in the shutter glasses.
\end{abstract}

\section{Introduction}

The last year a number of 3DTV sets have been introduced to the market by almost all the major TV manufacturers. The preferred solution for presenting stereoscopic 3D has been to use shutter glasses. With the increase of the number of systems, the need to characterize their performance in a consistent and meaningful way increases.

Crosstalk is, as defined by Seigel (2001)[1], the electrical or optical mixing of left- and right-eye image channels. Crosstalk is according to Meesters et al (2004) [2] probably one of the main perceptual factors contributing to perceived image quality and visual comfort. They also pointed out that crosstalk is highly dependent upon the type of stereoscopic system used. For autostereosopic displays, it has also been shown that certain amounts of crosstalk may be desirable to reduce ghosting and view flipping[1,3] but as pointed out by Lambooij et al[3] the optimal amount of crosstalk for those types of displays is still an issue of debate. In this paper, we are focusing on stereoscopic displays.

The Video Quality Experts Group (VQEG) is a group of experts from various backgrounds and affiliations, including participants from several internationally recognized standardization organizations, working in the field of video quality assessment [8]. One of their ad-hoc projects is targeting 3D video quality. The current plan is the performing of a multi-laboratory perceptual crosstalk video quality experiment. As part of the effort it is important to physically measure the crosstalk characteristics of the display systems in the different laboratories. The plan is to use mainly display systems with shutter glasses, which will be also in the focus of this paper. The goal is to specify a practical measurement procedure that will produce consistent results across laboratories.

In this paper, we first identify sources of crosstalk on shutterglasses systems based on continuous temporal measurements. Then we describe a crosstalk measurement method using averaging calibrated luminance meters. The consistency of this method is finally tested during a measurement campaign involving two different display models in three different labs.

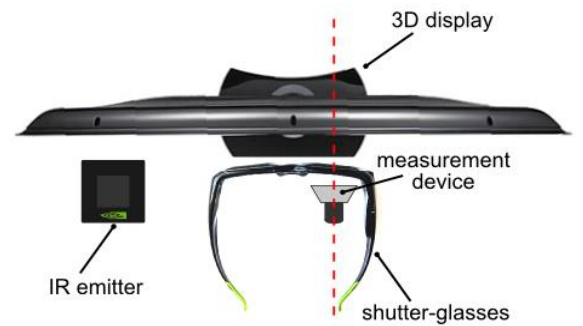

Figure 1: Top view of the measurement set-up.
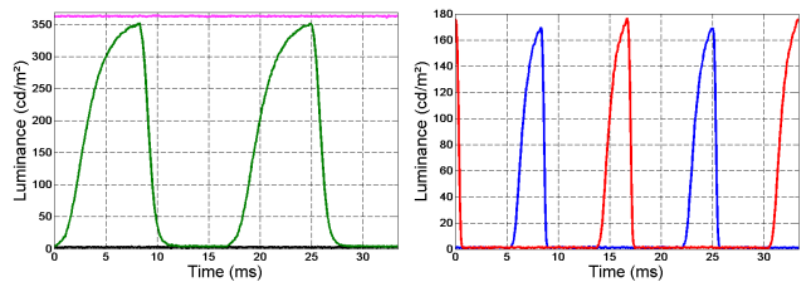

Figure 2: Examples of temporal waveforms measured directly on the display (left) and through the shutter-glasses (right). See text for more details.

\section{Temporal Crosstalk Measurement}

In order to identify crosstalk and understand which parameters affect it, we have performed temporal luminance measurements of the display alone as well as through the shutter-glasses, using the set-up as shown in Figure 1. The high sampling rate of these measurements permited us to characterize the temporal transmittance of the glasses and to synchronize it with the temporal response of the display. This knowledge was then used to identify the potential sources of crosstalk and to determine a measurement set-up and protocol using usual luminance-meters.

The temporal luminance measurements were performed with a Burr-Brown OPT101 monolithic photo-diode with an on chip trans-impedance amplifier. It has a fast response $(28 \mu \mathrm{s}$ from $10 \%$ to $90 \%$, rise or fall time). The signal was read by an oscilloscope with a sampling period of $20 \mu$ s (sample frequency of $50 \mathrm{kHz}$ ). When measuring the display luminance directly, the photo-diode was positioned in contact with the screen surface. When measuring the luminance through one glass, the photodiode and the glasses were put together and positioned as close to screen as possible.

Signals used were RGB signal with the same value for the three channels in each frame. In the rest of this section, signals will be identified by $L n R m$, where $n$ is the gray value of the left view and $m$ the gray value of the right view. Figure 2 presents some examples of waveforms. On the left plot, L0R0 (black), L0R255 (green) and L255R255 (magenta) have been measured directly on the display. On the right plot, $L 255 R 0$ has been measured through the left glass (red) and L0R255 has been measured through the right glass (blue). 


\subsection{Shutter Glasses Measurements}

\subsubsection{Synchronization}

The shutter-glasses of the Nvidia 3D Vision system used in this paper were synchronized with the display using a dedicated USB IR emitter. By measuring simultaneously the luminance emitted by the display and the luminance passing through the glasses, we have been able to synchronize display and glasses waveforms. The same temporal axis (thus reflecting this synchronization) has been used on Figure 2 left and Figure 2 right.

It can be observed that the glasses shutter is synchronized with respect to the end of the display frame period. More precisely, the glass is switched off when the luminance emitted by the display begins to change. It can also be observed that the opening of the glasses is quite short, with duration at half maximum of about $2.6 \mathrm{~ms}$ (one third of the frame period). Thus the glasses are open when the update of the displays pixel is supposed to be finished and when the display luminance is the highest and the closest to the desired final level.

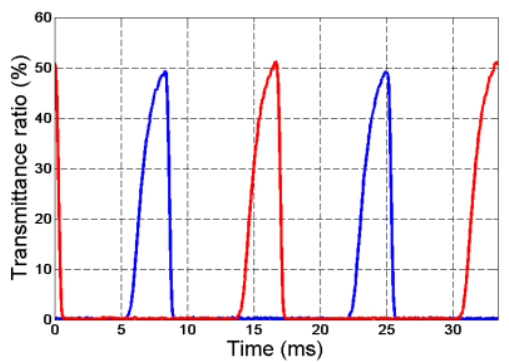

Figure 3: Transmittance functions of both left (red) and right (blue) eye-glasses.

\subsubsection{Transmittance}

The temporal transmittance of the glasses is defined as the ratio of luminance which passes through the glasses as a function of time. It can be estimated from the measurements as follows:

$$
T_{l}=\frac{M_{R 255 L 255}^{l}-M_{R 0 L 0}^{l}}{M_{R 255 L 255}^{d}-M_{R 0 L 0}^{d}}, \quad T_{r}=\frac{M_{R 255 L 255}^{r}-M_{R 0 L 0}^{r}}{M_{R 255 L 255}^{d}-M_{R 0 L 0}^{d}}
$$

where $T_{l}$ and $T_{r}$ are the transmittance functions of left and right shutters respectively, and $M_{s i g}^{a}$ identifies the waveform measured through left glass (if $a=l$ ), through right glass (if $a=r$ ), or directly on the display (if $a=d$ ), while the screen displayed the signal identified at the subscript position. For example $M_{R 255 L 255}^{l}$ is the waveform measured through the left glass while the screen displayed signal L255R255 (white frames on both views). Figure 3 illustrates both transmittance functions.

From these temporal transmittance waveforms, two transmittance ratios can be calculated. The transmittance of the glass when open $\alpha$ is estimated as the average value of the waveform in a one-display-frame wide rectangular window centered on the peak. The transmittance of the glass when closed $\beta$ is estimated as the average value of the waveform in the rest of the period. Table 1 gives the values for both glasses.

The glass-dependent crosstalk factor can be identified with $\beta$.

Table 1: Glass transmittance when open $(\alpha)$ and when closed $(\beta)$.

\begin{tabular}{|c|c|c|}
\cline { 2 - 3 } \multicolumn{1}{c|}{} & $\alpha$ & $\beta$ \\
\hline Right glass & $\alpha_{r}=12.2 \%$ & $\beta_{r}=0.23 \%$ \\
\hline Left glass & $\alpha_{l}=12.7 \%$ & $\beta_{l}=0.25 \%$ \\
\hline
\end{tabular}

\subsection{Display Measurements}

It can be noticed from the measurements of signal L0R255 (Figure 2 left, green waveform) that within the short frame period of the display in 3D mode $(120 \mathrm{~Hz})$ the final level of luminance is not reached. Since the gray-to-gray transition from 0 to 255 is known to be one the fastest rising transition on LC displays, it can be expected that the desired level of luminance will not be reached in most of the cases, as previously stated by Shestak et al. [6] and Boher et al. [7]. This can lead to a difference between the desired and actual luminance.

In order to quantify this problem, we measured two sets of signals: $L x R 0$ and $L x R x$, with $x$ varying from 0 to 255 with a step of 5 . The luminance reached for each view was directly measured on the display, and determined as the average of the luminance in the last third of the display frame period (i.e. when the glasses are open). Figure 4 illustrates these results.

It can be observed a large difference in reached luminance depending on whether the two views were identical or different. In this example, the difference was up to $-20 \%$ in the left view and $+180 \%$ in the right view. It is not clear at this point if this difference is a drawback due to the slow response time of the display or if it is a feature (at display side or at drivers side) to compensate or reduce glass-dependent crosstalk.
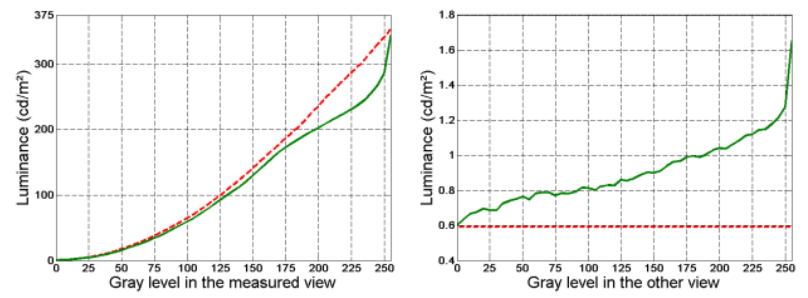

Figure 4: Luminance reached by the left view (green left) and by the right view (green right) for signal $L x R 0$, compared with luminance of signals $L x R x$ (red left) and $L 0 R 0$ (red right)

\subsection{Conclusion: Crosstalk Factors}

These first measurements have permitted to highlight two different factors of crosstalk: 1) a glass-dependent factor and 2) a display-dependent factor. The first can be simply measured and estimated for one given pair of goggles. The second one is more subject to caution since it involves inter-view dependency and is then hard to predict. It has to be noted that time average measurements, as those presented in the following, do not permit to differentiate influence from these different factors.

\section{Time Average Measurements}

This method follows a similar set-up as illustrated in Figure 1Error! Reference source not found.. Differences reside in 1) the measurement device, and 2) the procedure used during measurements. These points are described below.

The measurements were performed at the three different labs of the authors. Depending on the measurement device used, different distances between the display and the glasses were used. In particular, IRCCyN used a measurement device meant for measuring close to the screen while Mid Sweden University (MIUN) and Acreo used distant pointing devices. Furthermore, care was taken to make sure that the measurement device meter stayed fixed throughout the different measurements as the luminance of the screen may be position dependent. 
Table 2: Measurement conditions in the three labs

\begin{tabular}{|l|l|l|l|}
\cline { 2 - 4 } \multicolumn{1}{c|}{} & \multicolumn{1}{c|}{ IRCCyN } & \multicolumn{1}{c|}{ MIUN } & \multicolumn{1}{c|}{ Acreo } \\
\hline Manufacturer & CRS: Optical & KonicaMinolta & Photo Research \\
\& Model & OP200-E & LS-110 & PR522/524 \\
\hline Measuring angle & 13 degree & 0.5 degree & 1 degree \\
\hline Measurement area & $261 \mathrm{~mm}^{2}$ & $72 \mathrm{~mm}^{2}$ & $40 \mathrm{~mm}^{2}$ \\
\hline Dist. screen-glass & $50 \mathrm{~mm}$ & $1000 \mathrm{~mm}$ & $260 \mathrm{~mm}$ \\
\hline Dist. glass-device & $30 \mathrm{~mm}$ & $100 \mathrm{~mm}$ & $150 \mathrm{~mm}$ \\
\hline
\end{tabular}

Care was also taken to make sure that no external light reached the sensor, e.g. from ambient light sources and reflections. The different lab conditions are summarized in Table 2.

Two different displays combined with PC systems equipped with NVIDIA 3D vision (active shutter glasses) have been used in this study and they are:

- $\quad$ Alienware Optx AW2310 (1920x1080 pixels)

- $\quad$ Samsung SyncMaster 2233RZ (1680x1050 pixels)

The measurement method has been proposed for the inter laboratory crosstalk measurements for VQEG's crosstalk experiment, which will be described here.

\subsection{Stimuli}

A specifically written software player for 3D video, able to play uncompressed 3D video, has been used. Video sequences were prepared in native display resolution for each display.

The sequences were stored using a 4:2:2 YUV format, see eg. [4]. Three different video sequences were prepared. In each sequence and for each given time, the complete screen showed a single color (gray) value. The only exception was a small text area on the upper left corner which showed textual information on the stimulus presented. As only gray values were used, the two color difference components $\mathrm{Cb}$ and $\mathrm{Cr}$ were set to their neutral value 128 .

Each video sequence was preceded with a synchronization sequence of $\mathrm{Y}=0$ followed by $\mathrm{Y}=255$, another $\mathrm{Y}=0$ and $\mathrm{Y}=255$. Each of these four stimuli was displayed for 4 frames at a frame rate of $1 / 10^{\text {th }}$ of a second resulting in a presentation time of 40 seconds. In each frame the text "INTRO" was displayed in the upper left corner. This procedure of starting the sequence was used for synchronization of the measurement device and for checking on whether the measurement device reacted fast enough and was stabilized during the measurement cycle. All video sequences are available from the authors.

Sequence 1 (SEQ1): This sequence contained a stairstep of different luma values $(Y)$. The left view and the right view show the same content. The stairstep started with a value of $\mathrm{Y}=0$, $\mathrm{U}=128, \mathrm{~V}=128$. It proceeded with $\mathrm{Y}=90, \mathrm{U}=128, \mathrm{~V}=128$. The number "90" was displayed as a text in the upper left corner. Next, the Y component took sequentially the values $15,105,30$, $120, \ldots$ (The procedure of alternating $0,90,15,105, \ldots$ helped to manually read the measurement device as the step of 90 introduced a clear change of the displayed value).

Sequence 2 (SEQ2): This sequence contained the same stairstep as explained for SEQ1 except that only the left view changed. The right view stayed at $\mathrm{Y}=0$ all the time until the stairstep signal had finished once. Then another stairstep was displayed on the left view while the right view had the fixed value $Y=80$. This second luma value of the crosstalking side was chosen in order to stabilize the results for non-zero crosstalk values. The value 80 was chosen arbitrarily. This second value was meant for verification purpose as some display systems include a crosstalk reduction system and thus behave differently in function of the luminance on the second view. A value of $Y=80$ is sufficiently high to show such effects while not stressing the luminance measurement range when compared to $\mathrm{Y}=0$.

Sequence 3 (SEQ3): This sequence contained exactly the same sequence as SEQ2 except that the views were exchanged.

\subsection{Measurement procedure}

In the first measurement, no eye-glasses were used. SEQ1 was displayed and the obtained average luminance values from the photometer were stored.

For the following measurements, the eye-glasses were placed in front of the screen and well aligned with the luminance sensor. The measurement of SEQ1 was performed with the sensor looking through the left eye-glass. The same was then performed with SEQ2 as well as SEQ3, after which it was repeated for the right eye-glass.

\subsection{Crosstalk Factor Calculation}

The crosstalk factor was calculated independently for the left and the right eye. For shutter glasses, there are at least two sources of crosstalk. The first source of crosstalk is insufficient shadowing when the eye-glasses are switched to opaque mode. The second source of crosstalk is a temporal mismatch between the image shown on the screen and the opening of the shutter glasses. This also includes the effect that the LCD display might be reacting too slowly to a change in luminance between the two views.

In total, there are three sources of luminance which are captured by the human eye: the luminance which is meant to be shown, the luminance which crosstalks from the other view and the luminance which stems from insufficient isolation of the backlight of the display. The latter is the luminance which can be measured even if the display is showing a signal with $\mathrm{Y}=0$.

Thus, the perceived luminance on the right view is written as:

$$
L_{p}^{r}=L_{d}^{r}+L_{c}^{l}+L_{b}
$$

with $L_{p}^{r}$ corresponding to the perceived luminance on the right view which is the sum of $L_{d}^{r}$, the desired luminance on the right view, $L_{c}^{l}$ the crosstalking luminance that originates from the left view, and $L_{b}$ which is the background luminance of the display. Obviously, the crosstalk luminance $L_{c}^{l}$ is a factor of the signal displayed on the other view. The simplest way of modeling is to assume a multiplicative factor for the displayed signal, e.g.

$$
L_{c}^{l}=C_{l} \cdot L_{d}^{l}
$$

with $C_{l}$ being the crosstalk factor and $L_{d}^{l}$ being the desired luminance on the left view.

The crosstalk factor for the left view $C_{l}$ and the right view $C_{r}$ can be determined graphically by plotting the results of the measurement described earlier as

$$
C_{l}=\frac{M_{S E Q 2}^{r}-\min \left(M_{S E Q 2}^{r}\right)}{M_{S E Q 2}^{l}-\min \left(M_{S E Q 2}^{l}\right)} \quad C_{r}=\frac{M_{S E Q 3}^{l}-\min \left(M_{S E Q 3}^{l}\right)}{M_{S E Q 3}^{r}-\min \left(M_{S E Q 3}^{r}\right)}
$$

In this equation a vector of crosstalk coefficients $C_{l}$ is obtained which is calculated from the measurement obtained with SEQ2 and the sensor looking through the right glass for $M_{S E Q 2}^{r}$ and through the left glass $M_{S E Q 2}^{l}$. Each value in this vector corresponds to one measured luma point, e.g. $Y=0,15, \ldots, 255$. 


\section{Results and Discussion}

It has been seen that these crosstalk values are not stable at low luminance levels which might be an artifact due to the noise of the luminance measurement device. In particular, for the measurements from Acreo, the measurement device was unable to capture low luminance values. In order to avoid clipping artifacts at the highest luminance values, it should therefore be considered best practice to use the mean (or median) value of the crosstalk factor between $\mathrm{Y}=105$ and $\mathrm{Y}=225$. Figure 6 (a-b) presents the crosstalk factors obtained for the stable range of luminance values. The mean values are presented in Error! Reference source not found.

It can be observed that the values differ largely between the left and the right eye glasses. The difference between the IRCCyN and the MIUN labs are similar to those. Overall, the measured crosstalk factor is very small, being less than $1 \%$ in all cases.

Table 3: Average crosstalk factors in per mill for $\mathrm{Y}=0$

\begin{tabular}{|c|c|c|c|c|}
\hline \multirow{2}{*}{} & \multicolumn{2}{|c|}{ Alienware } & \multicolumn{2}{c|}{ Samsung } \\
\cline { 2 - 5 } & left $C_{l}$ & right $C_{r}$ & left $C_{l}$ & Right $C_{r}$ \\
\hline IRCCyN & 6.8 & 7.9 & 6.4 & 5.6 \\
\hline MIUN & 4.5 & 6.2 & 6.2 & 4.9 \\
\hline Acreo & 1.7 & 3.1 & & \\
\hline
\end{tabular}

The second measurement with the inactive view being $\mathrm{Y}=80$ revealed that this measurement depends strongly on the fact that no further processing in the display drivers, graphics card or display is activated. The results for the measurements on both displays in the three labs are provided in Figure 6 (c-d).

In contrast with previous results the crosstalk factor depends strongly on the Y-value at which it was measured. At low luminance values, the crosstalk is slightly larger than $1 \%$ but negative values are calculated for higher Y-values. In Figure 5, the cause of these negative values can be noticed clearly as for higher luminance values in the left view, the right view is considerably lowered. This is probably due active crosstalk cancellation in one of the display components, for example the overdrive technology used in the display. This could have a similar effect as was already discussed in Section 2.2 but this time the display seems to be overcompensating the temporal response instead of reacting too slowly to the luminance change.

\section{Conclusions}

The main purpose of this study was to define a measurement method which is easily reproducible from one lab to another and which gives results with no significant differences. It can be observed from Table 3 that there are some discrepancies in the results between the different labs but also between the eye glasses. Moreover, the temporal measurement presented in the

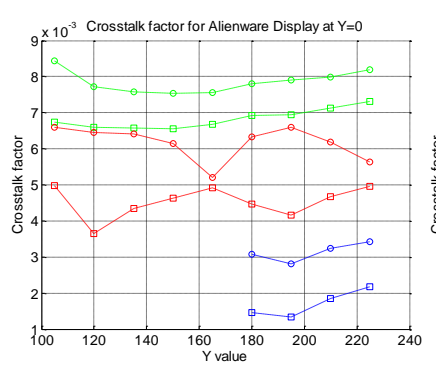

(a)

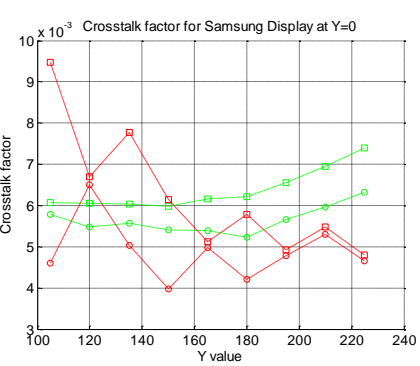

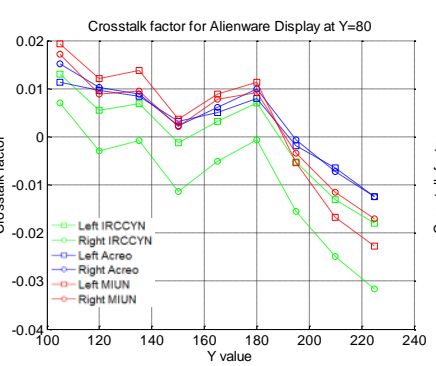

(c) first part of the paper leads to different results. This may be due to differences in the calibration of measurement devices, as well as in the experiment set-up.

It has been shown that many influence factors need to be considered, starting from the measurement setup and the different measurement devices. It was also seen that the display chain may introduce unpredictable effects and that crosstalk might need to be measured at several different gray levels. Work is still in progress in three different labs to find a suitable experiment set-up that fits any measurement device, in order to minimize inter-lab differences.
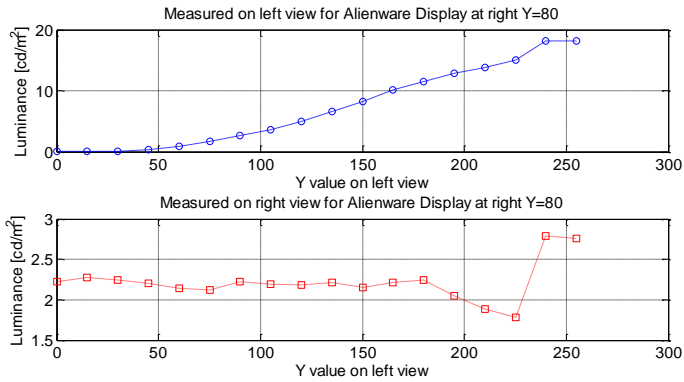

Figure 5: Measured luminance through the left and right eye-glass for constant luminance on the right view $\mathrm{Y}=\mathbf{8 0}$.

\section{References}

[1] Siegel, M., "Perceptions of Crosstalk and the Possibility of a Zoneless Autostereoscopic Display", Proc. of SPIE, Stereoscopic Displays and Virtual Reality Systems (2001)

[2] Meesters, L. et al., "A Survey of Perceptual Evaluations and Requirements of Three-Dimensional TV", IEEE Tran. on Circuits and Systems for Video Technology 14 (2004)

[3] Lambooij, M. et al., "Visual Discomfort and Visual Fatigue of Stereoscopic Displays: A Review", Journal of Imaging Science and Technology 53 (2009)

[4] Poynton, C., Digital Video and HDTV Algorithms and Interfaces, Morgan Kaufmann, Elsevier Science (2003)

[5] Pan, C. et al., "10.3: Cross-Talk Evaluation of ShutterType Stereoscopic 3D Display", SID Symp. Digest (2010)

[6] Shestak, S. et al. "10.4: Measuring of Gray-to-Gray Crosstalk in a LCD Based Time-Sequential Stereoscopic Display", SID Symp. Digest (2010)

[7] Boher, P. et al., "Optical Characterization of Shutter Glasses Stereoscopic 3D displays", SPIE Conference on Stereoscopic Displays and Applications (2011)

[8] Video Quality Experts Group (VQEG), Homepage, http://www.vqeg.org, (2010).

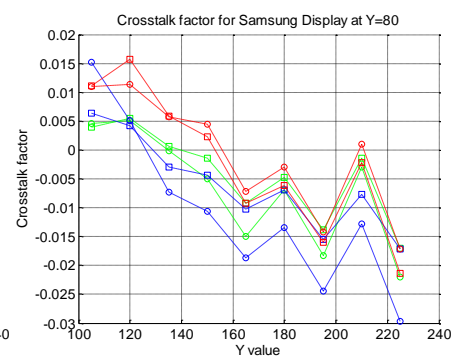

(d)

Figure 6: Crosstalk factors for both conditions and both display models in the stable range of $Y=105$ to $Y=225$. 